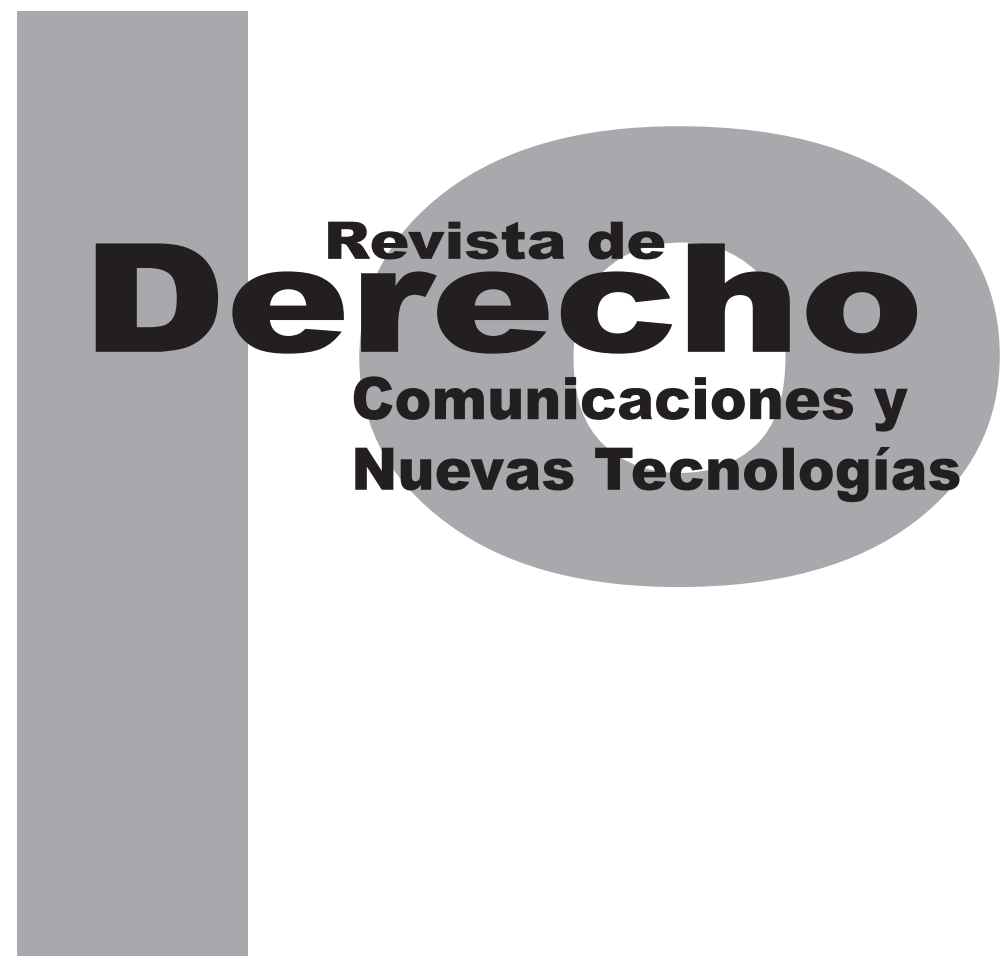

\title{
Obligatoriedad JURídica en MÉXICO de lOS INSTRUMENTOS INTERNACIONALES DE PROTECCIÓN DE DATOS PERSONALES
}

\author{
RIgOBERTO MARTíNeZ BECERRIL
}

\section{Artículo de reflexión}

DOI: http://dx.doi.org/10.15425/redecom.13.2015.03

Universidad de los Andes

Facultad de Derecho

Revista de Derecho, Comunicaciones y Nuevas Tecnologías

No.13, Enero - Junio de 2015. ISSN 1909-7786 


\section{Obligatoriedad jurídica en México de los instrumentos internacionales de protección de datos personales}

\section{Resumen}

El presente ensayo, a través del método deductivo busca demostrar la obligatoriedad que en el sistema jurídico mexicano pueden tener los diversos instrumentos jurídicos internacionales que regulan la protección de los datos personales, al amparo del principio de supremacía constitucional y el mecanismo de control de convencionalidad, tomando en consideración que solo los tratados internacionales celebrados en los términos que establece la Constitución Política de los Estados Unidos Mexicanos son obligatorios.

Palabras clave: Constitución, supremacía constitucional, control de convencionalidad, jerarquía normativa, tratados internacionales, datos personales, obligatoriedad.

\section{Legal Obligation in Mexico of International Instruments for Personal Data Protection}

\section{Abstract}

This essay, through the deductive method, aims to demonstrate the requirement that in the Mexican legal system may have the different international legal instruments governing the protection of personal data under the principle of constitutional supremacy and conventionality control, considering that only international treaties concluded under the terms established by the Political Constitution of the Mexican United States are required.

Keywords: Constitution, constitutional supremacy, conventionality control, law hierarchy, international treaties, personal data, obligation.

\section{Obrigatoriedade jurídica dos instrumentos internacionais de proteção de dados pessoais no México}

\section{Resumo}

O presente ensaio, através do método dedutivo busca demostrar a obrigatoriedade que no sistema jurídico mexicano podem ter os diversos instrumentos jurídicos internacionais que regulam a proteção dos dados pessoais, ao amparo do princípio de supremacia constitucional e o mecanismo de controle de convencionalidade, tomando em consideração que só os tratados internacionais celebrados nos termos que estabelece a Constituição Política dos Estados Unidos Mexicanos são obrigatórios.

Palavras-chave: Constituição, supremacia constitucional, controle de convencionalidade, hierarquia normativa, tratados internacionais, dados pessoais, obrigatoriedade. 


\title{
Obligatoriedad jurídica en México de los instrumentos internacionales de protección de datos personales*
}

\author{
Rigoberto Martínez Becerril ${ }^{* *}$
}

\begin{abstract}
SUMARIO
Introducción - I. CONCEPTO DE JERARQUÍA NORMATIVA Y SUPREMACÍA CONSTITUCIONAL - II. INTERPRETACIONES DE LA SUPREMA CORTE DE JUSTICIA DE LA NACIÓN AL ARTÍCULO 133 DE LA CONSTITUCIÓN POLÍTICA DE LOS ESTADOS UNIDOS MEXICANOS - III. CONTROL DE CONVENCIONALIDAD - IV. ORGANISMOS INTERNACIONALES - A. Organización de las Naciones Unidas (onu) - B. Consejo de Europa - C. Organización para la Cooperación y el Desarrollo Económicos (OCDE) - D. Foro de Cooperación Económica Asia-Pacífico (APEC) - E. Red Iberoamericana de Protección de Datos (RIPD) - V. INSTRUMENTOS JURÍDICOS INTERNACIONALES DE PROTECCIÓN DE DATOS PERSONALES, IMPORTANTES PARA MÉXICO - VI. CONCLUSIONES - Referencias.
\end{abstract}

\footnotetext{
* Cómo citar este artículo: Martínez Becerril, R. (Junio, 2015). Obligatoriedad jurídica en México de los instrumentos internacionales de protección de datos personales. Revista de Derecho, Comunicaciones y Nuevas Tecnologías, 13. Universidad de los Andes (Colombia).

** Licenciado en Derecho por la Universidad Nacional Autónoma de México. Ha ocupado diversos cargos en la Administración Pública Federal de México, actualmente se desempeña como Director de Proyectos y Coordinación del Instituto Federal de Telecomunicaciones. Autor del libro El derecho de acceso a la información pública en México, su ejercicio y medios de impugnación y de artículos para las revistas El Mundo del Abogado y AS Asesores en Soluciones. Correo: martinez.rigoberto89@yahoo.com ,Twitter: @RigobertoMtzB.
} 
Introducción

Ernesto Rey Cantor (2008), en su obra Control de convencionalidad de las leyes y derechos humanos, expone de manera magistral diversos temas de vanguardia en el ámbito internacional que inciden de manera directa y forzosa en principios e instituciones del derecho interno, como por ejemplo en el "principio de supremacía constitucional".

La supremacía constitucional es un tema que ha sido abordado por muchos autores desde hace siglos y cuya concepción, influida por diversos factores de carácter político, económico, jurídico y social, ha evolucionado de manera constante.

La vigente Constitución Política de los Estados Unidos Mexicanos consagra la supremacía constitucional en el artículo 133, artículo que en su redacción original (Constitución de 1917) establecía como Ley Suprema de la Unión "todos los tratados hechos y que se hicieran por el Presidente de la República".

En 1934, el referido artículo 133 constitucional fue modificado para reconocer ese mismo carácter a "todos los tratados que estén de acuerdo con la misma [Constitución]". La razón de esta enmienda obedeció a que la Constitución establecía, desde un principio, que los tratados de carácter internacional solo vincularían al país a partir de su ratificación por el Senado, y en los términos en que estaba redactado originalmente el artículo 133, se daba una marcada incongruencia entre la atribución del Senado para ratificar los tratados internacionales y convenciones diplomáticas celebradas por el Ejecutivo (artículo 76, fracción I) con la potestad según la cual las solas decisiones del Presidente de la República podrían constituirse en Ley Suprema de toda la Unión (Valadés, 1979, pág. 182).

"La reforma de este artículo es más al texto que a su contenido", se dijo en el dictamen de la Cámara de Senadores; pero la reforma implicó una modificación de fondo. Antes de la reforma, la Suprema Corte de Justicia estimó que un tratado internacional debe ser aplicado no obstante que disponga algo contrario a la Constitución, y ahora posiblemente no le quede más remedio que negar la obligatoriedad de un tratado internacional anticonstitucional (Valdez, 1945, págs. 443-444).

Durante los últimos años, la Constitución Política de los Estados Unidos Mexicanos ha sufrido una serie de cambios importantísimos, entre los que se destacan los contenidos en el Decreto publicado en el Diario Oficial de la Federación, del 10 de junio de 2011, misma que en palabras de la Suprema Corte de Justicia de la Nación:

Evidencia el reconocimiento de la progresividad de los derechos humanos, mediante la expresión clara del principio pro persona como rector de la interpretación y aplicación de las normas jurídicas, en aquellas que favorezcan y brinden mayor protección a las personas. Así, la ampliación de los derechos que significa la concreción de algunas cláusulas constitucionales, como aquella relativa a los migrantes o a la suspensión de garantías, aunada a la obligación expresa de observar los tratados 
internacionales firmados por el Estado mexicano, miran hacia la justiciabilidad y eficacia de los derechos que, a la postre, tiende al mejoramiento de las condiciones de vida de la sociedad y al desarrollo de cada persona en lo individual (párr. 3).

La reforma en cita modificó el artículo $1^{\circ}$ constitucional, introduciendo el denominado "control de convencionalidad" mediante el cual se obliga a las autoridades del Estado mexicano a realizar, en el ámbito de sus competencias, una revisión de congruencia entre las normas nacionales y las internacionales, y a resolver los asuntos sometidos a su consideración utilizando la norma más favorable, en estricto apego al principio pro homine, cuando estén en juego derechos humanos, poniendo incluso, a dicho de varios, en crisis el principio de supremacía constitucional.

Bajo esa óptica, en el presente ensayo se abordarán los temas de jerarquía normativa, el principio de supremacía constitucional, el control de convencionalidad y el análisis de la naturaleza jurídica de los instrumentos internacionales más importantes en materia de protección de datos personales, y mediante la utilización del método deductivo se buscará determinar si dichos instrumentos internacionales son de carácter obligatorio en el sistema jurídico mexicano, lo cual resulta de suma importancia respecto de las controversias que se susciten en torno a la protección de los datos personales, en tanto que nos delimita el marco jurídico aplicable.

\section{CONCEPTO DE JERARQUÍA NORMATIVA Y SUPREMACÍA CONSTITUCIONAL}

El tema del orden jerárquico normativo es abordado desde la Edad Media con San Agustín y Santo Tomás, filósofos cristianos cuyas teorías tienen como punto de partida a Dios, pues sostienen que todo nace en Él y ha de retornar a Él.

Ambos afirman la idea de un Dios trascendente y creador del universo. Dios, al crear el mundo, le imprimió un orden natural, cada cosa tiene una esencia y por lo tanto un fin propio.

Santo Tomás de Aquino establece una estrecha relación entre ley y razón. Incluso llega a la identificación de la causa por la cual el obrar racionalmente o actuar humano deriva de la naturaleza misma del hombre, diciendo que ello es una facultad que reside en la esencia de toda persona.

Santo Tomás da autonomía a la fe de la razón, porque según señalaba, la ley estaba compuesta por la razón y la voluntad divina, poniendo el acento por igual en ambas facultades. Cada persona tiene inmersa en su interior la ley eterna y al ser nosotros seres racionales podemos conocerla moviendo a la voluntad humana para que cumpla dicha ley, afirmaba.

Para los dos, la ley adopta diversas formas dependiendo de dónde provenga, y llegan a establecer un orden jerárquico en el que colocan en primer lugar a la ley eterna, puesto que proviene directamente de Dios; en segundo lugar, como 
reflejo de la primera, en los seres humanos encontramos la ley natural y la ley física en los seres irracionales; en tercer lugar, como una manifestación del derecho positivo, encontramos la ley humana o ley temporal, como la llama San Agustín.

Otros de los análisis del orden jerárquico normativo, y seguramente uno de los más conocidos, es el realizado por el célebre jurista, político y filósofo austriaco Hans Kelsen, en su obra Teoría pura del Derecho.

Kelsen comienza su análisis con la fundamentación de validez de una norma, la cual considera que se encuentra en otra de jerarquía superior: "El fundamento de validez de una norma sólo puede encontrarse en la validez de otra norma. La norma que representa el fundamento de validez de otra es caracterizada, metafóricamente, como una norma superior en relación con una inferior" (1986, pág. 201).

Sin embargo, el sostener que la fundamentación de una norma debe encontrarse forzosamente en otra superior, nos llevaría al extremo necesario de una escala normativa infinita, lo cual es resuelto por Kelsen estatuyendo una norma suprema, la que desde luego no debe estar subordinada a ninguna otra norma. Al respecto manifiesta:

Como norma suprema tiene que ser presupuesta, dado que no puede ser impuesta por una autoridad cuya competencia tendría que basarse en una norma aún superior. Su validez no puede derivarse ya de una norma superior, ni puede volver a cuestionarse el fundamento de su validez. Una norma semejante, presupuesta como norma suprema, será designada aquí como norma fundante básica (Kelsen, 1986, pág. 202).

La norma fundante básica a que refiere Kelsen es la Constitución:

El instrumento escrito que ha convenido el pueblo (...) como la norma absoluta de acción y decisión para todos los departamentos y funcionarios del Gobierno (...) y en oposición a la cual cualquier ley o norma de cualquier departamento o funcionario del Gobierno o aún del pueblo mismo, serán completamente nulos (Cooley, citado por Schwartz, 1966, pág. 24).

Por tanto, la Constitución es fuente común de validez de todas las normas pertenecientes a un mismo sistema u orden jurídico; el hecho de que muchas normas encuentren su fundamento o validez en una misma norma fundante básica, ocasiona que pertenezcan o conformen un mismo sistema u orden normativo, con las diferentes materias de las cuales se integra.

Otra de las características de las normas, la cual le da pertenencia a determinado sistema normativo, es que esas normas deben producirse conforme a la norma fundante básica.

Aquello que constituye la unidad de un orden jurídico es la idea de una norma fundamental que presuponga hipotéticamente todo conocimiento jurídico. Esta norma representa la validez de las normas que pertenecen a un mismo orden jurídico, pero no determina su contenido, sino las facultades para su creación. 
Al ser la Constitución el punto de partida del orden normativo, esta representa la unidad y armonía a seguir por el sistema jurídico, lo que se traduce en el principio de fundamentalidad constitucional, mediante el cual la Constitución debe guardar en sí misma un equilibrio de principios generales y excepciones a ellos, con la finalidad de que el sistema normativo que de ella se desprende sea un todo armonizado.

Al desprenderse el sistema normativo de la Constitución, que en sí misma no guarda contradicción, las normas que de ella se derivan no deben rebasar lo estipulado por el texto constitucional. Es por ello que al explicar la estructura lógica de diversos ámbitos normativos dados, Kelsen llega no solo a la construcción escalonada del orden mismo, sino a estudiar las relaciones entre estos órdenes, manifestando que de existir supuestas contradicciones entre ellos, estas son resueltas mediante la interpretación del orden jurídico establecido, de tal manera que el derecho tenga una totalidad plena de sentido (Soto, 2012, págs. 154-156).

Con base en los esbozos anteriores, autores recientes como Humberto Suárez Camacho nos dicen:

La jerarquización de las leyes se constituye por la relación de supra y subordinación en que se encuentran las normas dentro de un orden jurídico dado, porque, por virtud de la Constitución del Estado, su sistema de derecho deja de ser una coordinación de normas, como sucede en el derecho internacional y en el derecho primitivo, también llamado por la doctrina formalista como consuetudinario.
Así la estructuración de las normas jurídicas parte de la generalidad a la concreción; la norma original, producto genuinamente político, se traduce en directriz jurídica del resto de los ordenamientos derivados de ella, los cuales no pueden apartarse de sus postulados.

El principio de jerarquía normativa produce las siguientes consecuencias, relacionadas tanto con la organización jurídica estatal como al control de la constitucionalidad y legalidad que derivan de su aplicación:

A. UNIDAD.- A través de la jerarquización de normas se asegura la compatibilidad vertical y horizontal de las normas en un ordenamiento jurídico. Así, las normas inferiores deben adecuarse a las superiores y las normas de igual nivel no deben contradecirse o contraponerse.

B. CONTROL.- La armonía en la producción de ordenamientos secundarios, respetando las reglas de las leyes jerárquicamente superiores o equivalentes, hace innecesaria la tarea de la compatibilidad entre las normas. La posible ruptura de la unidad se produce, ordinariamente, cuando en la creación o aplicación de normas abstractas, surge el cuestionamiento del emisor o el contenido mismo de la ley, que, al ser una creación humana, es susceptible de error, mismo que debe ser subsanado por el órgano constitucionalmente establecido para verificar el ajuste de la norma controvertida con la unidad y la supremacía constitucional, para así mantener la efectividad de todo el sistema jurídico.

C. RAZONABILIDAD.- La finalidad de las leyes consiste en ser instrumentos o medios adecuados para la realización de los fines que establezca la Constitución; es decir, la producción del univer- 
so normativo secundario surge de la necesidad de complementar las prevenciones constitucionales que, para conseguir realidad, requieren la coexistencia de leyes, si bien menores en jerarquía, con una mayor vitalidad. Esto ha llevado a parte de la doctrina a señalar a la Constitución más como un mandato de legislar que una norma de aplicación estricta en controversias particulares. Sin embargo, la racionalidad, -conjuntamente con el sub principio de control-dan dinámica a la norma constitucional, al asignarle valor directriz que no puede ser vulnerado por el legislador secundario.

c. RIGIDEZ CONSTITUCIONAL.- Se ha visto, bajo el aspecto formal de Constitución, la necesidad de distinguir entre el Poder Constituyente, creador de la norma original, y los poderes constituidos, a través del establecimiento de una distribución formal-funcional de competencias dirigidas a determinar quiénes deben crear los distintos niveles jurídicos. Asimismo, se formaliza o dificulta la reforma constitucional, pues peligraría el principio de supremacía constitucional si la Constitución pudiese ser reformada 0 adicionada mediante los procedimientos legislativos ordinarios (2007, págs. 24-26).

Así las cosas, me atrevo a definir la jerarquía normativa como la ordenación escalonada de las normas jurídicas, que tiene como propósito evitar que las normas de rango inferior contradigan o vulneren lo establecido por una norma de rango superior, normas todas que sin importar su rango deben estar alineadas y ser concordantes con la norma fundamental, que se constituye como la norma jurídica de más alto estrato.
En otro orden de ideas, la jerarquía normativa se encuentra íntimamente relacionada con la supremacía constitucional, la cual:

Debe entenderse como: aquella cualidad que posee únicamente la Constitución como norma jurídica, al ser el punto de partida de legitimidad de todo el orden jurídico de un país o territorio determinado.

La Constitución, es producto de un acto soberano del pueblo para instituirla como Carta Fundamental de un orden jurídico, es por ello que implica dos condiciones que son: la de Poder Constituyente como portador de la voluntad soberana del pueblo y en consecuencia autor de la Constitución; y como producto de este, los Poderes Constituidos, los cuales adquieren sus facultades de un documento superior constitutivo de ellos, que es la Constitución.

La Constitución es suprema por ser la expresión de la voluntad soberana del pueblo, realizada a través del Congreso o Asamblea Constituyente, la cual, es fuente $u$ origen de los poderes que crea y organiza; los mismos, no pueden ir más allá de su norma creadora, lo que implica que sea superior a ellos.

La Supremacía es necesaria porque se requiere de una norma fundamentadora de todas las demás leyes y normas conformadoras del orden jurídico, esa es la Constitución; ella obliga a todos los demás ordenamientos a seguirla en su contenido, a no contravenirla, a respetarla por ser la ley de mayor jerarquía. Una Constitución es suprema por ser fundamental y es fundamental por ser suprema.

La Constitución se encuentra por encima del Estado, por encima de los órganos constituidos 
y por encima de los individuos considerados aisladamente, puesto que como el pueblo, al ser el titular de la soberanía, está por encima de la propia Constitución que en el artículo 39 le reconoce el inalienable derecho de alterar o modificar la forma de su gobierno, conforme a su voluntad y atendiendo los intereses que estime convenientes.

La Constitución, como cúspide de todo ordenamiento jurídico, hace fluir el principio de legalidad en los poderes públicos, de seguridad jurídica de los actos de autoridad, de constitucionalidad en todas las actuaciones realizadas por los poderes constituidos (Soto, 2012, págs. 131 y 132).

Bajo ese orden de ideas, la Constitución, que es obra y creación del Poder Constituyente Originario (Negri y De Marco, 1994, pág. 18):

Se significa por ser un compendio de normas jurídicas que paralelamente a su naturaleza deontológica, abrigan la pretensión de regular y encauzar los procesos ontológicos que brotan y se manifiestan en la sociedad. La Constitución, entonces, es ley; precisamente por esta característica, el principio de supremacía que se complementa con el de fundamentalidad dice que la Constitución es ley suprema y, por serlo, es también fundamental (Uribe, 2010, pág. 50).

Sin embargo, Uribe afirma que poco se podría agregar a la apreciación referida en el párrafo inmediato anterior. "El resultado de esta supremacía es que el orden jurídico, en su totalidad, se encuentra, de alguna manera, sometido a la Constitución y que ninguna autoridad del Estado tiene poderes o facultades por fuera de la Constitución" (Tamayo, 1989, pág. 235).

\section{INTERPRETACIONES DE LA SUPREMA CORTE DE JUSTICIA DE LA NACIÓN, AL ARTÍCULO 133 DE LA CONSTITUCIÓN POLÍTICA DE LOS ESTADOS UNIDOS MEXICANOS}

El artículo 133 de la Carta Magna, producto de la evolución y constante cambio de la realidad social, política, económica y jurídica, ha variado a lo largo de los años en la interpretación que le ha dado el órgano máximo del Poder Judicial, aspecto que se abordará brevemente en los siguientes párrafos. Para empezar, comenzaremos por citarlo:

Artículo 133. Esta Constitución, las leyes del Congreso de la Unión que emanen de ella y todos los Tratados que estén de acuerdo con la misma, celebrados y que se celebren por el Presidente de la República, con aprobación del Senado, serán la Ley Suprema de toda la Unión. Los jueces de cada Estado se arreglarán a dicha Constitución, leyes y tratados, a pesar de las disposiciones en contrario que pueda haber en las Constituciones o leyes de los Estados.

La interpretación doctrinal y jurisprudencial del dispositivo constitucional en estudio, por mucho tiempo fue constante en mantener la concepción monista de supremacía del orden jurídico original sobre los tratados internacionales y, desde luego, respecto de las leyes constitucionales y demás ordenamientos jurídicos que, en su expedición y aplicación, establecen el deber de ajustarse a los designios de la norma fundamental, como puede observarse en la siguiente tesis: 
TRATADOS INTERNACIONALES, AMPARO CONTRA LA APLICACIÓN DE LOS. No debe sobreseerse en el juicio de amparo, por la causa de improcedencia que establece la fracción XVIII del artículo 73 de la Ley de Amparo, en relación con el artículo 133 de la Constitución General de la República, pues aun cuando los tratados internacionales celebrados por el presidente de la República, con aprobación del Senado, que estén de acuerdo con la propia Constitución, son, junto con esta y con las leyes del Congreso de la Unión, que emanan de ella, la Ley Suprema de toda la Unión, ni el precepto constitucional contenido en el artículo 133 ni otro alguno de la propia Carta Fundamental o de la Ley de Amparo, proscriben el juicio de garantías contra la indebida aplicación de un tratado, ya que es indudable que los actos que las autoridades administrativas realizan para cumplimentar tratados internacionales, deben estar debidamente fundados y motivados $y$ originarse en un procedimiento en el que se hayan Ilenado las formalidades que señala la misma Constitución, pues una actitud distinta pugna abiertamente con el artículo 14 de la citada Carta Magna. En esas condiciones, si el juicio de amparo es el medio de control de la legalidad de los actos de autoridad, debe estimarse procedente aunque se trate de la aplicación de tratado internacional, ya que de lo contrario se dejaría en estado de indefensión al particular afectado (scjn Segunda Sala. Tesis aislada (común). J. irráñitu. Semanario Judicial de la Federación: xcvill, agosto 1965, pág. 61).

Respecto del tópico de mérito, relacionado con la problemática de control surgida por la suscripción de tratados internacionales, en nuestro ámbito jurídico aparece la relación jerárquica que guardan las leyes federales con los tratados internacionales. Sobre este particular, había sido criterio de la Suprema Corte de Justicia de la Nación (en adelante scın) que las leyes federales y los tratados internacionales tenían la misma jerarquía normativa entre sí, tal y como se desprende de la tesis jurisprudencial siguiente:

\section{LEYES FEDERALES Y TRATADOS INTERNACIONA-} LES. TIENEN LA MISMA JERARQUIA NOR-

MATIVA. De conformidad con el artículo 133 de la Constitución, tanto las leyes que emanen de ella, como los tratados internacionales, celebrados por el ejecutivo Federal, aprobados por el Senado de la República y que estén de acuerdo con la misma, ocupan, ambos, el rango inmediatamente inferior a la Constitución en la jerarquía de las normas en el orden jurídico mexicano. Ahora bien, teniendo la misma jerarquía, el tratado internacional no puede ser criterio para determinar la constitucionalidad de una ley ni viceversa. Por ello, la Ley de las Cámaras de Comercio y de las de Industria no puede ser considerada inconstitucional por contrariar lo dispuesto en un tratado internacional (scjn Pleno. Tesis aislada (Constitucional). V. Adato. Semanario Judicial de la Federación: 60, diciembre de 1992, pág. 27).

La tesis anterior se basa únicamente en una interpretación literal del artículo 133 constitucional, sin distinguir el carácter constitucional u ordinario de la ley federal ni la época de expedición de esta en relación con el tratado internacional.

Cabe destacar que la scın abandonó el criterio anterior, optando por aquel que dispuso que los tratados internacionales estén jerárquicamente por encima de las leyes federales, aunque por debajo de la Constitución Federal: 
TRATADOS INTERNACIONALES. SON PARTE INTEGRANTE DE LA LEY SUPREMA DE LA UNIÓN Y SE UBICAN JERÁRQUICAMENTE POR ENCIMA DE LAS LEYES GENERALES, FEDERALES Y LOCALES. INTERPRETACIÓN DEL ARTÍCULO 133 CONSTITUCIONAL. La interpretación sistemática del artículo 133 de la Constitución Política de los Estados Unidos Mexicanos permite identificar la existencia de un orden jurídico superior, de carácter nacional, integrado por la Constitución Federal, los tratados internacionales y las leyes generales. Asimismo, a partir de dicha interpretación, armonizada con los principios de derecho internacional dispersos en el texto constitucional, así como con las normas y premisas fundamentales de esa rama del derecho, se concluye que los tratados internacionales se ubican jerárquicamente abajo de la Constitución Federal y por encima de las leyes generales, federales y locales, en la medida en que el Estado Mexicano al suscribirlos, de conformidad con lo dispuesto en la Convención de Viena Sobre el Derecho de los Tratados entre Ios Estados y Organizaciones Internacionales o entre Organizaciones Internacionales y, además, atendiendo al principio fundamental de derecho internacional consuetudinario "pacta sunt servanda", contrae libremente obligaciones frente a la comunidad internacional que no pueden ser desconocidas invocando normas de derecho interno y cuyo incumplimiento supone, por lo demás, una responsabilidad de carácter internacional (scjn Pleno, Tesis aislada (Constitucional), S.S. Aguirre. Semanario Judicial de la Federación: XXV, abril de 2007, pág. 6).

Sobre este mismo tópico, la scsn ha seguido emitiendo criterios jurisprudenciales, obligada por los conflictos que en torno a los derechos humanos se han suscitado:
TRATADOS INTERNACIONALES. CUANDO LOS CONFLICTOS SE SUSCITEN EN RELACIÓN CON DERECHOS HUMANOS, DEBEN UBICARSE A NIVEL DE LA CONSTITUCIÓN. Los tratados O convenciones suscritos por el Estado mexicano relativos a derechos humanos, deben ubicarse a nivel de la Constitución Política de los Estados Unidos Mexicanos, porque dichos instrumentos internacionales se conciben como una extensión de lo previsto en esa Ley Fundamental respecto a los derechos humanos, en tanto que constituyen la razón y el objeto de las instituciones. Por lo que los principios que conforman el derecho subjetivo público, deben adecuarse a las diversas finalidades de los medios de defensa que prevé la propia Constitución y de acuerdo con su artículo 133 las autoridades mexicanas deben respetarlos, por lo que bajo ninguna circunstancia pueden ser ignorados por ellos al actuar de acuerdo a su ámbito competencial.

PRIMER TRIBUNAL COLEGIADO EN MATERIAS ADMINISTRATIVA Y DE TRABAJO DEL DÉCIMO PRIMER CIRCUITO.

AMPARO DIRECTO 1060/2008. 2 de julio de 2009. Mayoría de votos. Disidente: Hugo Sahuer Hernández. Ponente: Juan García Orozco. Secretario: Víctor Ruiz Contreras.

Nota: Esta tesis fue objeto de la denuncia relativa a la contradicción de tesis 293/2011, resuelta por el Pleno el 3 de septiembre de 2013 (scjn Tribunales colegiados del Circuito. Tesis aislada (común), J. García. Semanario Judicial de la Federación: XXXI, mayo 2010, pág. 2079).

Por último, resulta sumamente importante para nuestro tema en estudio hacer referencia a lo expuesto por la scın al resolver la Contradicción de Tesis 293/2011. 
A mayor abundamiento, la contradicción en cita analizó como uno de los temas motivo de la enunciada contradicción, la posición jerárquica de los tratados internacionales en materia de derechos humanos frente a la Constitución.

Al respecto, el Séptimo Tribunal Colegiado en Materia Civil del Primer Circuito estableció que derivado de la tesis: "TRATADOS INTERNACIONALES. SE UBICAN JERÁRQUICAMENTE POR ENCIMA DE LAS LEYES FEDERALES Y EN SEGUNDO PLANO RESPECTO DE LA CONSTITUCIÓN FEDERAL" establecida por el Tribunal Pleno, los tratados internacionales en materia de derechos humanos, se ubicaban jerárquicamente por debajo de la Constitución.

Por otra parte, el Primer Tribunal Colegiado en Materia Administrativa y de Trabajo del Décimo Primer Circuito, señaló que "cuando se trate de un conflicto que verse sobre derechos humanos, los tratados o convenciones internacionales suscritos por el Estado Mexicano, deben ubicarse propiamente a nivel de la Constitución". De tal posicionamiento derivó la siguiente tesis: “TRATADOS INTERNACIONALES. CUANDO LOS CONFLICTOS SE SUSCITEN EN RELACIÓN CON DERECHOS HUMANOS, DEBEN UBICARSE A NIVEL DE LA CONSTITUCIÓN".

Al respecto, el Máximo Tribunal, por mayoría de diez votos, sostuvo que existe un reconocimiento en conjunto de derechos humanos cuyas fuentes son la Constitución y los tratados internacionales de los cuales el Estado mexicano es parte.

Además, se estableció que de la interpretación literal, sistemática y originalista del contenido de las reformas constitucionales de 6 y 10 de junio de 2011, se desprende que las normas de derechos humanos, independientemente de su fuente, no se relacionan en términos jerárquicos, sin embargo, cuando la Constitución establezca una restricción expresa al ejercicio de los derechos humanos, se deberá estar a lo que indica la norma constitucional. En este sentido, los derechos humanos, con independencia de su fuente, constituyen el parámetro de control de regularidad constitucional, conforme al cual debe analizarse la validez de todas las normas y actos de autoridad que forman parte del ordenamiento jurídico mexicano (scjn Pleno. Contradicción de Tesis 293/2011, párr. 14).

En ese orden de ideas, con base en los diversos criterios emitidos por la scJn puedo afirmar que el principio de supremacía constitucional en México no es obsoleto, pues desde mi perspectiva la Constitución Política de los Estados Unidos Mexicanos sigue estableciendo como norma fundante básica el orden jerárquico normativo en el sistema jurídico nacional, respecto del cual, como se ha visto, ha colocado a los tratados internacionales; entendidos estos como el convenio regido por el derecho internacional público, celebrado por escrito entre el Gobierno de los Estados Unidos Mexicanos y uno o varios sujetos de derecho internacional público, ya sea que para su aplicación requiera o no la celebración de acuerdos en materias específicas, cualquiera que sea su denominación, mediante el cual los Estados Unidos Mexicanos asumen compromisos, con la acotación que estos deberán ser aprobados por el Senado y serán Ley Suprema de toda la Unión, de acuerdo con el artículo $2^{\circ}$ de la Ley sobre Celebración de Tra- 
tados (DOF, 2 de enero de 1992), a la par de los dispositivos constitucionales, cuando estos contengan derechos humanos.

En otras palabras, si en un tratado suscrito por el Presidente de los Estados Unidos Mexicanos, debidamente ratificado por el Senado, se reconocen derechos humanos que de forma expresa no se consagran en el texto constitucional, dichos derechos deberán considerarse con el mismo nivel jerárquico de un derecho que se contemple en la Constitución Política de México, es decir, los derechos humanos que se contengan en los tratados internaciones suscritos por el Estado mexicano que cumplan con todos los requisitos que exige la Constitución para su validez pasarán a formar parte del catálogo de derechos humanos del derecho doméstico mexicano, con rango constitucional.

\section{CONTROL DE CONVENCIONALIDAD}

Rey Cantor (2008, págs. 46 y 47) establece que el control de convencionalidad puede ser en sede internacional o en sede nacional.

En sede internacional es un mecanismo de protección procesal que ejerce la Corte Interamericana de Derechos Humanos (Corte IDH), en los casos en que el derecho interno - sea la Constitución, los actos administrativos, la jurisprudencia, las prácticas administrativas o judiciales, etc.-, resulte incompatible con la Convención Americana sobre Derechos Humanos (CADH) u otros tratados. Para ello, la Corte $\mathrm{IDH}$ mediante un examen de confrontación normativo en un caso concreto, dicta una sentencia judicial ordenando la modificación, derogación, anulación o reforma de las normas o prácticas internas, según corresponda, protegiendo los derechos humanos, con el objeto de garantizar la supremacía de la CADH.

En sede nacional, el control de convencionalidad se presenta cuando el juez interno tiene competencia para inaplicar el derecho interno y aplicar la CADH u otro tratado, luego del examen de confrontación normativo, en un caso concreto, y adopta una decisión judicial protegiendo los derechos de la persona humana.

Aunado a lo anterior, Ruiz (1997) manifiesta que el control de convencionalidad puede operar de dos formas:

1.- Juicio de Convencionalidad por "acción" del legislador: "es posible que el tribunal declare que la existencia de una determinada legislación pueda generar la violación de alguno de los derechos reconocidos en el Convenio" (pág. 39).

2.- Juicio de Convencionalidad por la "omisión" del legislador: "Lo que viola un derecho reconocido en el Convenio no es tanto la existencia de una legislación como su inexistencia" (p. 39).

La promulgación de normativa incompatible con las obligaciones internacionales de un Estado, o bien, la falta de legislación necesaria para el cumplimiento de dichas obligaciones genera incurrir en responsabilidad internacional por parte del Estado-Legislador. 
De acuerdo con Charles Rousseau (citado en Rey Cantor), la responsabilidad internacional del Estado-Legislador puede darse como consecuencia de acción o de abstención por parte del órgano legislativo.

a) Hay responsabilidad por acción en caso de promulgarse una ley contraria a las obligaciones internacionales de un Estado;

b) Hay responsabilidad por omisión en dos supuestos: cuando el órgano legislativo no dicta las leyes necesarias para el cumplimiento de las obligaciones internacionales del Estado y cuando no deroga una ley contraria a dichas obligaciones (2008, pág. เv).

Lo brevemente comentado en torno al control de convencionalidad da una idea general de su importancia y trascendencia en la teoría jurídica moderna, y el impacto que tiene la suscripción de tratados y convenciones: asumir y cumplir una serie de compromisos cuya exigibilidad se puede alcanzar a través de los diversos mecanismos que van generando tanto el sistema jurídico internacional como el sistema jurídico nacional.

El control de convencionalidad se vislumbraba desde hace varios años, dada la existencia de ciertas normas consuetudinarias de derecho internacional referidas a la aplicación en el ámbito interno de los Estados, aceptadas por la comunidad internacional, y plasmadas inclusive en diversos criterios jurisprudenciales, entre las que destacan:

1.- Un Estado no puede hacer prevalecer su derecho interno para incumplir una obligación internacional, es decir, un Estado no puede alegar contra otro Estado su propia Constitución con el fin de eludir obligaciones que le incumben en virtud del derecho internacional o tratados vigentes.

2.- El derecho interno es un simple "hecho" para el derecho internacional, entendiendo por "hecho" las manifestaciones de la voluntad o actividad del Estado, al igual que las decisiones judiciales o las medidas administrativas.

3.- El Estado que asume un compromiso internacional debe adecuar su legislación interna para cumplir con el tratado o con el convenio suscrito.

4.- Las disposiciones de derecho interno no pueden prevalecer sobre las de un tratado.

Todo lo anterior generó la construcción de un nuevo orden jerárquico del derecho interno en relación con el derecho internacional, a partir del conflicto que implicaba determinar cuál de los dos primaba sobre el otro. Esto originó diversos supuestos atendiendo a distintas hipótesis, a saber:

a) En el caso que el conflicto sea entre una norma de derecho internacional y otra de derecho interno y dicho conflicto se produzca dentro del ámbito internacional como, por ejemplo, ante un tribunal internacional, la preeminencia de la norma de derecho internacional es admitida sin discusión alguna.

b) Si el conflicto entre una norma de derecho internacional y una de derecho interno se presen- 
ta dentro del ámbito interno de un Estado y es una autoridad judicial o administrativa nacional la llamada a resolverlo, esta deberá, ciertamente, aplicar lo que su correspondiente derecho interno disponga al respecto, específicamente su Constitución.

c) Para Edmundo Vargas Carreño, los tratados internacionales pueden prevalecer aun respecto a la Constitución del Estado.

d) En el ordenamiento jurídico interno existe la posibilidad de que los tratados y convenios internacionales, o el derecho internacional general tenga rango constitucional, ni superior ni inferior a la Constitución.

e) En algunas Constituciones se prescribe que las normas de derecho internacional tienen un valor superior a las leyes internas.

El conflicto consistente en determinar qué debe prevalecer cuando el derecho internacional y el derecho interno se confrontan ha dado paso a una serie de posiciones diversas, que sin duda ponen en entredicho instituciones y principios bastante arraigados en la doctrina y en la práctica jurídica de muchas naciones, entre ellos sin duda el principio de supremacía constitucional, más aún con la aparición, consolidación y cada vez mayor reconocimiento del control de convencionalidad, pero sobre todo ante el surgimiento de controversias que pueden afectar en grado superlativo los derechos humanos y cuya solución exige el apoyo de instrumentos jurídicos internacionales.
En México, la scsn ha aclarado:

El párrafo segundo del artículo 10. de la Constitución Política de los Estados Unidos Mexicanos dispone que las normas relativas a los derechos humanos se interpretarán de conformidad con la propia Constitución y con los tratados internacionales de la materia favoreciendo en todo tiempo a las personas la protección más amplia, de donde deriva que los tribunales federales, en los asuntos de su competencia, deben realizar el estudio y análisis ex oficio sobre la constitucionalidad y convencionalidad de las normas aplicadas en el procedimiento, o en la sentencia o laudo que ponga fin al juicio. Ahora bien, esta obligación se actualiza únicamente cuando el órgano jurisdiccional advierta que una norma contraviene derechos humanos contenidos en la Constitución Federal o en los tratados internacionales de los que el Estado Mexicano sea parte, aun cuando no haya sido impugnada, porque con su ejercicio oficioso se garantiza la prevalencia de los derechos humanos frente a las normas ordinarias que los contravengan (scjn Segunda Sala. Jurisprudencia (común). Semanario Judicial de la Federación: junio 27 de 2014, pág. 555).

Lo anterior permite ver el estatus de privilegio en el que nuestra Carta Magna coloca a los derechos humanos, al grado de permitir que las autoridades del Estado mexicano acudan a la interpretación de la Constitución y de los tratados internacionales para brindar una protección más extensa.

De la concepción anterior surge el denominado Principio pro homine, el cual "implica que ante diferentes interpretaciones de un dispositivo legal, debe optarse por aquella que conduzca a 
una mejor y más amplia protección de los derechos fundamentales, descartando así las que restrinjan o limiten su ejercicio". ${ }^{2}$

Bajo ese entendido, se gesta la obligación de las autoridades del Estado mexicano de analizar los actos de autoridad no solo a la luz del texto constitucional, sino además al amparo de los derechos humanos contenidos en los tratados internacionales celebrados por México en los términos exigidos por la Carta Magna para su validez; así como para que en caso de que exista una diversidad de interpretaciones se utilice aquella que más favorezca a la persona, independientemente del fundamento que se utilice, es decir, Constitución o tratado, o bien, que se adopte la interpretación que menos limite el ejercicio de sus derechos humanos.

\section{ORGANISMOS INTERNACIONALES}

Para efectos del presente ensayo, resulta importante hacer alusión a algunos de los organismos internaciones más influyentes a nivel mundial, entre estos, la Organización de Naciones Unidas (ONU), el Consejo de Europa, la Organización para la Cooperación y el Desarrollo Económicos (OCDE), el Foro de Cooperación Económica AsiaPacífico y la Red Iberoamericana de Protección de Datos.

Semanario Judicial de la Federación. "Principio pro homine o pro persona. Si en un caso concreto no se actualiza la antinomia de dos normas que tutelan derechos humanos para que el juzgador interprete cuál es la que resulta de mayor beneficio para la persona, aquél no es el idóneo para resolverlo." Décima Época, Tribunales Colegiados de Circuito, Libro 3, febrero de 2014, Tomo III, pág. 2019.

\section{A. Organización de las Naciones Unidas}

Según se lee en la página web de la onu:

Las Naciones Unidas son una organización internacional fundada en 1945 tras la Segunda Guerra Mundial por 51 países que se comprometieron a mantener la paz y la seguridad internacionales, fomentar entre las naciones relaciones de amistad y promover el progreso social, la mejora del nivel de vida y los Derechos Humanos (s.f., pág. 1).

Su instrumento jurídico fundacional es la Carta de las Naciones Unidas, la cual se firmó el 26 de junio de 1945 en San Francisco, al terminar la Conferencia de las Naciones Unidas sobre Organización Internacional, y entró en vigor el 24 de octubre del mismo año.

La Carta de las Naciones Unidas establece los propósitos de la onu, entre estos: mantener la paz y la seguridad internacionales; fomentar entre las naciones relaciones de amistad basadas en el respeto al principio de la igualdad de derechos y al de la libre determinación de los pueblos; realizar la cooperación internacional en la solución de problemas internacionales de carácter económico, social, cultural o humanitario, y en el desarrollo y estímulo del respeto a los derechos humanos y a las libertades fundamentales de todos, sin hacer distinción por motivos de raza, sexo, idioma o religión; y servir de centro que armonice los esfuerzos de las naciones por alcanzar estos propósitos comunes.

Para el cumplimiento de tales propósitos, los miembros de la onu se conducen bajo ciertos 
principios contenidos en el artículo 2 de la Carta de las Naciones Unidas, de los cuales y para efectos de nuestro tema resultan destacables los siguientes:

a) Cumplimiento de buena fe de las obligaciones contraídas por sus miembros;

b) La solución de sus controversias internacionales por medios pacíficos; $y$,

c) La prohibición para que en la Carta de las Naciones Unidas se establezca la autorización a las Naciones Unidas para intervenir en los asuntos que son esencialmente de la jurisdicción interna de los Estados, ni para obligar a los miembros a someter dichos asuntos a procedimientos de arreglo conforme a la enunciada Carta; sin perjuicio de la aplicación de las medidas coercitivas que se tomen por el Consejo de Seguridad en contra de un Estado por amenazas a la paz o actos de agresión.

Así las cosas, es posible aseverar que la onU basa sus acuerdos en la buena fe de sus miembros, en la solución pacífica de sus conflictos y en el no intervencionismo, salvo en los casos en los que el Consejo de Seguridad se vea obligado a tomar medidas por actos que amenacen la paz mundial.

\section{B. Consejo de Europa}

El Consejo de Europa es una organización internacional que nace tras la segunda Guerra Mundial, el 5 de mayo de 1949 luego de la firma de su Carta fundacional -el Tratado de Londres- por parte de Bélgica, Francia, Luxemburgo, Países Bajos y Reino Unido. Poco después, se adhirieron Irlanda, Italia, Dinamarca, Noruega y Suecia.

Este organismo internacional es el guardián de los valores democráticos en el continente europeo, tiene como objetivo principal la defensa y protección de la democracia, el Estado de Derecho y los derechos humanos, en particular los civiles y políticos.

Engloba a la totalidad de las naciones europeas con la sola excepción de Bielorrusia. Tiene su sede en la ciudad francesa de Estrasburgo y su órgano más activo es el Tribunal Europeo de Derechos Humanos.

La finalidad del Consejo de Europa, conforme a su Estatuto, consiste en generar una unión más estrecha entre sus miembros para salvaguardar y mover los ideales y los principios que constituyen su patrimonio común y favorecer su progreso económico y social.

Cada uno de los miembros del Consejo de Europa reconoce el principio del imperio del Derecho y el principio en virtud del cual cualquier persona que se halle bajo su jurisdicción ha de gozar de los derechos humanos y de las libertades fundamentales, y se compromete a colaborar sincera y activamente en la consecución de la finalidad mencionada en el párrafo anterior.

El artículo 4 del Estatuto del Consejo de Europa establece que cualquier Estado europeo, considerado capaz de cumplir el principio del imperio del derecho y de salvaguarda de derechos 
humanos de las personas que estén bajo su jurisdicción y que tenga voluntad de formar parte del enunciado organismo internacional, podrá hacerlo.

De lo anterior se advierte que el formar parte de este organismo internacional guardián de los valores democráticos en el continente europeo, tiene como objetivo principal la defensa y protección de la democracia, el Estado de Derecho y los derechos humanos; es un acto volitivo de buena fe, para cuyo ingreso es requisito sine qua non el respeto irrestricto del Estado de Derecho y la protección de los derechos humanos.

El Comité de Ministros es el órgano competente que actúa en nombre del Consejo de Europa, quien tiene la facultad de examinar, por recomendación de la Asamblea Consultiva o por iniciativa propia, las medidas adecuadas para realizar la finalidad de dicho Consejo, incluida la conclusión de convenios y de acuerdos, y la adopción por los gobiernos de una política común respecto a determinados asuntos. Sus conclusiones son comunicadas a los miembros por el secretario general, las que pueden revestir la forma de recomendaciones a los gobiernos.

Así las cosas, las decisiones de mayor calado por parte del órgano máximo del Consejo de Europa, pueden, en su caso, revestir la forma de recomendación, misma que será atendida por los miembros del Consejo, con plena libertad y atendiendo a su buena fe, aunque sabemos que existen siempre elementos extra jurídicos para el acatamiento de las decisiones por parte de los Estados.

\section{C) Organización para la Cooperación y el Desarrollo Económicos (OCDE)}

\begin{abstract}
Fundada en 1961, la Organización para la Cooperación y el Desarrollo Económicos (OCDE) agrupa a 34 países miembros y su misión es promover políticas que mejoren el bienestar económico y social de las personas alrededor del mundo.
\end{abstract}

La OCDE ofrece un foro donde los gobiernos puedan trabajar conjuntamente para compartir experiencias y buscar soluciones a los problemas comunes (OCDE, s.f., pág. 1)

La Convención de la Organización de Cooperación y de Desarrollo Económicos (en adelante la Convención) es el instrumento jurídico fundacional de esta organización internacional. Mediante ella se hace la reconstitución de la Organización Europea de Cooperación Económica, antecesora de la OCDE.

En el artículo I de la Convención se establecen los principales objetivos de la OCDE, los cuales se encauzan a la promoción de políticas tendientes a realizar la más fuerte expansión posible de la economía y del empleo y a un aumento del nivel de vida en los países miembros, manteniendo la estabilidad financiera y a contribuir así al desarrollo de la economía mundial; contribuir a una sana expansión económica en los países miembros y en los no miembros en vías de desarrollo económico; y contribuir a la expansión del comercio mundial sobre una base multilateral y no discriminatoria, conforme a las obligaciones internacionales. 
La Convención establece en su artículo V, que con el fin de alcanzar sus objetivos la Organización puede: tomar decisiones que, salvo dispuesto de otra forma, serán obligatorias para todos los miembros [cursivas añadidas], lo que desde luego resulta sumamente importante, pues denota el carácter imperativo que para sus miembros tienen las resoluciones de este organismo internacional, las cuales no serán vinculantes por excepción.

No obstante lo anterior, el diverso artículo VI de la Convención de mérito contempla que ninguna decisión será obligatoria para miembro alguno hasta que no haya sido incorporada a su ordenamiento jurídico conforme a las disposiciones de su procedimiento constitucional.

Así las cosas, la obligatoriedad de las resoluciones de la OCDE para sus miembros, deriva del artículo V de su Convención, sujeto a la condición de que se incorporen al sistema jurídico doméstico conforme a su orden constitucional, según lo dispone el artículo VI del instrumento jurídico de mérito.

\section{Foro de Cooperación Económica Asia-Pacífico (APEC)}

La idea del APEC, en primer lugar, fue abordada públicamente por el ex primer ministro de Australia, el Sr. Bob Hawke, durante un discurso en Seúl, Corea, en enero de 1989. Más tarde ese año, doce economías de Asia-Pacífico se reunieron en Canberra, Australia, para establecer el APEC. Los miembros fundadores fueron: Australia, Brunei Darussalam, Canadá, Indonesia, Japón, Corea, Malasia, Nueva Zelanda, Filipinas, Singapur, Tailandia y los Estados Unidos.
China, Hong Kong y el Taipei Chino se unieron en 1991, México y Papua Nueva Guinea siguieron en 1993. Chile se adhirió en 1994 y en 1998 se sumaron Perú, Rusia y Vietnam (APEC, s.f., pág. 1)

Así las cosas, APEC se integra actualmente por 21 países, foro de cooperación económica que entre 1989 y 1992 celebraba reuniones un tanto informales, hasta que en 1993, por propuesta del expresidente de los Estados Unidos de Norteamérica, se estableció como práctica la Reunión anual de líderes de APEC.

El Foro de Cooperación Económica Asia-PacífiCO (APEC, por sus siglas en inglés) se estableció en 1989 con el fin de aprovechar la creciente interdependencia de las economías de la región. APEC tiene como objetivo crear una mayor prosperidad para los habitantes de la región, fomentando un crecimiento económico inclusivo, equitativo, sustentable e innovador.

El peso económico de APEC es muy significativo: sus 21 miembros representan 54 por ciento del PIB mundial y 44 por ciento del comercio del mundo.

Los tres pilares de APEC Son: la liberalización del comercio y la inversión, facilitación para hacer negocios, y la cooperación técnica (Secretaría de Comercio. Gobierno de México, s. f.).

Este organismo internacional promueve la transparencia y el establecimiento de mejores prácticas en los procedimientos y reglamentos relacionados con el flujo de bienes, servicios y capital en la región de Asia-Pacífico, con la finalidad de generar confianza y certidumbre en los operadores económicos de la región. 
Finalmente, es de resaltar que: "APEC es un foro gubernamental de cooperación y consulta. Los objetivos de los mecanismos no contemplan la negociación de un TLC. APEC opera sin requerir que sus miembros celebren acuerdos con obligaciones legalmente vinculantes" (Secretaría de Comercio. Gobierno de México, 2012, pág. 3).

\section{E. Red Iberoamericana de Protección de Datos (RIPD)}

La Red Iberoamericana de Protección de Datos, surge con motivo del acuerdo alcanzado en el Encuentro Iberoamericano de Protección de Datos celebrado en La Antigua, Guatemala, en el año 2003, con la asistencia de representantes de 14 países iberoamericanos.

Esta iniciativa contó desde sus inicios con un apoyo político reflejado en la Declaración Final de la XIII Cumbre de Jefes de Estado y de Gobierno de los países iberoamericanos celebrada en Santa Cruz de la Sierra, Bolivia, 14 y 15 de noviembre de 2003, conscientes del carácter de la protección de datos personales como derecho fundamental, así como de la importancia de las iniciativas regulatorias iberoamericanas para proteger la privacidad de los ciudadanos.

La RIPD se configura como un foro integrador de los diversos actores, tanto del sector público como privado, que desarrollen iniciativas y proyectos relacionados con la protección de datos personales en Iberoamérica, con la finalidad de fomentar, mantener y fortalecer un estrecho y permanente intercambio de información, experiencias y conocimientos entre ellos, así como promover los desarrollos normativos necesarios para garantizar una regulación avanzada del derecho a la protección de datos personales en un contexto democrático, tomando en consideración la necesidad del continuo flujo de datos entre países que tienen diversos lazos en común y una preocupación por este derecho (RIPD, s.f.).

El instrumento jurídico que regula la RIPD es su Reglamento, con el que se institucionaliza, se fijan sus objetivos y se establece su régimen interno. El Reglamento de mérito establece derechos y obligaciones para sus miembros, al interior de la RIPD, que resultan necesarios y naturales a su participación, pero que no son vinculantes para los miembros.

No obstante lo anterior, el Reglamento de la RIPD prevé mecanismos de cooperación y cooperación reforzada, estos últimos generados a través de convenios de colaboración bilateral o multilateral entre su miembros, con los cuales se pretende que sobre la base del principio de reciprocidad y del pleno respeto al derecho de protección de datos de carácter personal, las instituciones y entidades miembros de la RIPD con competencias de ejecución y control promuevan el uso de instrumentos de cooperación para garantizar el cumplimiento efectivo de la normativa de protección de datos personales.

Con base en lo anterior podemos decir que si bien las determinaciones de la RIPD no son vinculantes, su Reglamento permite que sus miembros, a través de la celebración de convenios, adquieran compromisos para el efectivo cumplimiento de la normativa que rige la protección de datos personales. 


\section{INSTRUMENTOS JURÍDICOS INTERNACIONALES DE PROTECCIÓN DE DATOS PERSONALES, IMPORTANTES PARA MÉXICO}

El tema de la protección de los datos personales, el cual surge ante la necesidad de salvaguardar la información que sobre las personas obtienen, almacenan, transfieren y modifican tanto los órganos gubernamentales como los particulares, ha ocupado un lugar central en los foros, las aulas, los congresos y la agenda política de los Estados en los últimos años, incentivado por la constante evolución de los medios electrónicos. En cuanto a los organismos del Estado el acopio de datos deviene del ejercicio de sus atribuciones, y tratándose de los particulares, de la prestación de servicios y de la venta de productos.

El término habeas Data, como algún sector de la doctrina denomina al derecho para la protección de los datos personales, fue tomado parcialmente del antiguo instituto del habeas corpus, y su primer vocablo significa "conserva o guarda tu...", mientras que el segundo proviene del inglés data, sustantivo plural que significa "información o datos". En síntesis, en una traducción literal habeas data sería "conserva o guarda tus datos” (García, s.f., pág. 3).

Dicha prerrogativa ha sido interpretada de diferente manera por la doctrina especializada. Pérez Luño (1989) señala que el habeas data es un derecho fundamental de tercera generación, que tiene por objeto garantizar la facultad de las personas de conocer y acceder a las informacio- nes que les conciernen, archivadas en bancos de datos; controlar su calidad, lo que implica la posibilidad de corregir o cancelar los datos inexactos o indebidamente procesados y disponer sobre su transmisión.

Por su parte, Frosini (1988) se refiere a este como una nueva forma presentada por el derecho a la libertad personal, es decir, el derecho a controlar las informaciones sobre su propia persona.

Respecto de este mismo tema, Lucas Murillo define al habeas data como "el control que a cada uno de nosotros nos corresponde sobre la información que nos concierne personalmente, sea íntima o no, para preservar de este modo el último extremo, la propia identidad, nuestra dignidad y la libertad" (Enciclopedia Jurídica Mexicana, 2002, pág. 275).

En fin, independientemente del concepto que cada uno de nosotros nos formemos del derecho a la protección de datos personales, lo cierto es que los gobiernos del mundo se han ocupado de este tema que tiene un impacto mundial y en múltiples sectores, en gran medida, producto de los vehículos que permiten el tratamiento de los datos personales con más facilidad y con una mayor rapidez y, desde luego, porque los datos personales se han convertido a mi entender en el petróleo del ciberespacio.

Lo anterior se hace patente en la infinidad de instrumentos jurídicos que se han emitido con el propósito de ofrecer principios y parámetros mínimos para la salvaguarda y derechos irres- 
trictos de los datos personales, entre los que destacan los siguientes:

1.- La Resolución 509 del Consejo de Europa, del año 1968, la cual estudia la potencial agresividad que representan las tecnologías de la información para los derechos y libertades de las personas.

2.- Las Directrices de la Organización para la Cooperación y el Desarrollo Económicos (OCDE), del año 1980, que establecen los principios básicos aplicables al tratamiento de datos personales y a la vez garantizan la libre circulación de estos.

3.- El Convenio 108 del Consejo de Europa, del año 1981, que garantiza a los ciudadanos de los Estados contratantes el respeto de sus derechos y libertades, en particular, el derecho a la vida privada frente a los tratamientos de datos personales, conciliando el libre flujo de información entre los Estados contratantes.

4.- La Resolución 45/95 de la Asamblea General de la Organización de Naciones Unidas, de 1990, que enumera una serie de principios en materia de protección de datos personales de aplicación mundial.

5.- La Directiva 95/46/CE, la cual amplía los principios ya recogidos en otros instrumentos internacionales e impide la creación de barreras para la libre circulación de los datos personales en todos los Estados miembros de la Unión Europea.
6.- El Marco de Privacidad del Foro de Cooperación Económica Asia-Pacífico (APEC), del año 1999, mediante el cual se busca un equilibrio entre la seguridad de la información personal y el libre flujo de esta para fines comerciales.

7.- El artículo 8 de la Carta de Derechos Fundamentales de la Unión Europea, del año 2000, donde se reconoce el derecho a la protección de datos como un derecho fundamental y autónomo, distinto al derecho a la intimidad y a la privacidad de las personas (Ornelas, 2010).

8.- Directrices para la armonización de la protección de datos en la Comunidad Iberoamericana, expedidas por la Red Iberoamericana de Protección de Datos en el año 2007. Ofrecen a los poderes públicos de los Estados Iberoamericanos criterios orientativos para el desarrollo de iniciativas normativas en esta materia, facilitando el establecimiento de un marco homogéneo que favorezca el intercambio de los flujos de información entre los Estados y terceros Estados bajo estándares similares de protección.

Al respecto es menester aclarar que estos instrumentos jurídicos, pese a la importancia que poseen en la regulación del tema de protección de datos personales, representando sin duda un objeto de estudio mundial, no necesariamente tienen efectos imperativos en el sistema jurídico mexicano. Entre ellos, por supuesto, primeramente aquellos que rigen de forma exclusiva en un territorio determinado del cual México no es parte, como por ejemplo, la ResoIución 509 del Consejo de Europa; el Convenio 108 del Consejo de Europa, que si bien permite 
la anexión de otros países no europeos, los Estados Unidos Mexicanos no se han adherido a este; la Directiva 95/46/CE y el artículo 8 de la Carta de Derechos Fundamentales de la Unión Europea.

Por lo que respecta a la Resolución 45/95 de la Asamblea General de la Organización de Naciones Unidas, relativa a los principios rectores para la reglamentación de los ficheros computadorizados de datos personales, no le resulta legalmente vinculante a México, por cuanto estos son solo orientaciones para el establecimiento de las modalidades de aplicación de los reglamentos relativos a los ficheros computarizados de datos personales, los cuales se dejan a la libre iniciativa de cada Estado miembro.

En cuanto a las Directrices para la Armonización de la Protección de Datos en la Comunidad Iberoamericana, estas tampoco son coercitivas para México, pues su objetivo es ofrecer a los poderes públicos de los Estados Iberoamericanos criterios orientadores para el desarrollo de iniciativas normativas en esta materia, en la búsqueda de un marco jurídico homogéneo.

Por otra lado, y si bien es cierto México forma parte de la OCDE, sus Directrices relativas a la Protección de la Intimidad y de la Circulación Transfronteriza de Datos Personales constituyen solo una "recomendación" que busca armonizar las legislaciones nacionales de sus países miembros, relativas a la intimidad, y que a la vez que defiendan tales derechos, impidan interrupciones en la circulación internacional de datos que pudieran ocasionar graves trastornos en im- portantes sectores de la economía, tales como la banca y los seguros.

Finalmente, y por lo que respecta al Marco de Privacidad del Foro de Cooperación Económica Asia-Pacífico, este tampoco tiene una aplicación imperativa y obligatoria para los Estados Unidos Mexicanos pese a ser miembro de dicho foro. El objetivo del marco de privacidad en mención se limita a proporcionar una clara orientación y dirección a empresas dentro de las economías de APEC, sobre asuntos comunes de privacidad y del impacto de estos en la forma como se conducen negocios legítimos, destacando las expectativas razonables del consumidor moderno de que las empresas reconocerán sus intereses de privacidad de forma consistente con los principios explicados en dicho Marco.

No obstante lo anterior, el hecho de que los instrumentos jurídicos mencionados que rigen el derecho de protección de datos personales en el ámbito internacional no sean jurídicamente vinculantes, no quiere decir que México no los observa, pues su acatamiento puede acarrear ventajas o desventajas de índole económica, política, social, entre otras.

Por último, resulta prudente destacar que para el régimen jurídico mexicano, la Convención de la Organización de Cooperación y Desarrollo Económicos sí tiene el carácter de un tratado internacional, en los términos que prevé el artículo $2^{\circ}$ de la Ley sobre Celebración de Tratados, lo que implica que en un momento dado, la OCDE podría emitir disposiciones de carácter obligatorio para México, ya que así lo permite el inciso 
a), del artículo $V$ de la convención en cita. Lo anterior permitiría que las autoridades mexicanas, ante un eventual conflicto relacionado con los datos personales, tuvieran que aplicar el control de convencionalidad que ordena el artículo $1^{\circ}$ constitucional y el principio pro homine, sujeto a que, de acuerdo con el numeral VI de la Convención de la OCDE, tales disposiciones se incorporen al sistema jurídico mexicano conforme a su orden constitucional.

\section{CONCLUSIONES}

1.- La jerarquía normativa es la ordenación escalonada de las normas jurídicas, que tiene como propósito evitar que las normas de rango inferior contradigan o vulneren lo establecido por una norma de rango superior, normas todas que sin importar su rango deben estar alineadas y ser concordantes con la norma fundamental, que se constituye como la norma jurídica de más alto estrato.

2.- La norma jurídica de más alto estrato o norma fundante, misma que le da validez, legitimidad y pertenencia al resto de las normas que en ella se apoyan, es la Constitución, lo que permite sustentar el principio de supremacía constitucional.

3.- El principio de supremacía constitucional, consagrado en el artículo 133 de la Constitución Política de los Estados Unidos Mexicanos, se encuentra vigente, pues de la interpretación realizada en torno a este por la scjn, no se ha logrado establecer que exista una norma de rango superior a esta.
Lo anterior, no obstante la scjn haya interpretado que los derechos humanos contenidos en los tratados o convenciones suscritos por el Estado mexicano deben ubicarse a nivel de la Constitución Política. Ello obedece a la permisibilidad que se contiene en el artículo $1^{\circ}$ del propio texto constitucional.

4.- El artículo $1^{\circ}$ de la Constitución Política de los Estados Unidos Mexicanos consagra el control de convencionalidad, por medio del cual las autoridades mexicanas, en el ámbito de su competencia, tienen la obligación de interpretar de conformidad con la propia Constitución y con los tratados internacionales de la materia, aquellas normas que consagren derechos humanos, favoreciendo en todo tiempo a las personas la protección más amplia o bien, el menor perjuicio, lo que se concibe como principio pro homine.

5.- Para introducir una norma internacional a la infraestructura jurídica nacional de forma imperativa, esta debe tener el carácter de un tratado, esto es, ser un convenio regido por el derecho internacional público, celebrado por escrito entre el Gobierno de los Estados Unidos Mexicanos y uno o varios sujetos de derecho internacional público, cualquiera que sea su denominación, mediante el cual los Estados Unidos Mexicanos asuma compromisos, siempre y cuando sea ratificado por el Senado de la República.

6.- Ninguno de los instrumentos jurídicos internacionales que regulan de forma específica los datos personales, tales como la Resolución 509 del Consejo de Europa, las Directrices de la Organización para la Cooperación y el Desarrollo 
Económicos, el Convenio 108 del Consejo de Europa, la Resolución 45/95 de la Asamblea General de la Organización de Naciones Unidas, la Directiva 95/46/CE, el Marco de Privacidad del Foro de Cooperación Económica Asia Pacífico (APEC), el artículo 8 de la Carta de Derechos Fundamentales de la Unión Europea del año 2000 y las Directrices para la armonización de la protección de datos en la Comunidad Iberoamericana, cumplen en términos de la normativa mexicana con los elementos y formalidades para considerarlos tratados internacionales, por lo que, en consecuencia, no son jurídicamente vinculantes ni imperativos para México.

7.- Atendiendo a la naturaleza jurídica de la Convención de la Organización de Cooperación y Desarrollo Económicos, la cual para el Estado mexicano tiene el estatus de tratado internacional, la oCDE puede tomar decisiones en materia de protección de datos obligatorias para México, pero solo exigibles hasta que se hayan incorporado a su derecho doméstico conforme al procedimiento constitucional, según lo disponen los artículos V y VI de la Convención en cita.

8.- Finalmente, se puede concluir que pese a que diversos instrumentos jurídicos que regulan la protección de los datos personales en el ámbito internacional no posean el carácter de imperativos, ello no obsta para su cumplimiento por parte de México, pues estos generan ventajas de índole política, económica e inclusive social.
Referencias

Asia-Pacific Economic Cooperation. (s.f.). History. Recuperado de APEC: http://www.apec. org/About-Us/About-APEC/History.aspx

Estados Unidos Mexicanos. Secretaría de Gobierno. (s.f.). Foro de Cooperación Económica Asia-Pacífico (APEC). Obtenido de SE: http:// www.economia.gob.mx/comunidad-negocios/comercio-exterior/organismos-multilaterales/foro-de-cooperacion-economica-asiapacifico-apec

Frosini, V. (1988). Informática y Derecho. Bogotá, Colombia: Editorial Temis.

García Barrera, M. E. (s.f.). El Habeas Data en México. Recuperado de http://www.ctainl.org. $\mathrm{mx} /$ revista_7/noticias/habeasdatamexico. pdf

Gobierno de España. Ministerio de Asuntos Exteriores y de Cooperación (s.f.). Historia y actividad del Consejo de Europa. Obtenido de: http://www.exteriores.gob.es/Portal/es/ PoliticaExteriorCooperacion/ConsejoDeEuropa/Paginas/HistoriaActividadConsejoEuropa. aspx

Instituto de Investigaciones Jurídicas de la unam. (2002). Enciclopedia Jurídica Mexicana. México: Porrúa.

Kelsen, H. (1986). Teoría pura del Derecho, México, D. F.: Universidad Nacional Autónoma de México. 
Mclntyre Cooley, T. (1868). A Tratise on the Constitutional Limitations which Rest upons the Legislative Power of the States of the American Union. Boston, Mass.: Little, Brown and Co.

Naciones Unidas. (s.f.). La Organización. Obtenido de Naciones Unidas: http://www.un.org/ es/aboutun/

Negri, A. y De Marco, C. (1994). El poder constituyente. Ensayo sobre las alternativas de la modernidad. Madrid: Editorial Libertarias Prodhufi, S. A.

Organización para la Cooperación y el Desarrollo Económicos. (s.f.). ¿Qué es la oCDE? Recuperado de oCDE: http://www.oecd.org/centrodemexico/ laocde/

Ornelas Núñez, L. (Febrero de 2010). El estatus del derecho a la protección de datos personales en México. Congreso Internacional de Derecho y Tecnología, Universidad de Colima. Colima, México.

Pérez Luño, A. (1989). Los derechos humanos en la sociedad tecnológica. Cuaderno de Debates, (21). Centro de Estudios Constitucionales.

Presidencia de la República de México. Ley sobre Celebración de Tratados. Artículo 2o. Publicado en el Diario Oficial de la Federación el día 2 de enero del 1992.

Red Iberoamericana de Protección de Datos. (s.f.). Historia de la Red Iberoamericana. Re- cuperado de: http://www.redipd.org/la_red/ Historia/index-ides-idphp.php

Rey Cantor, E. (2008). Control de convencionalidad de las leyes y derechos humanos: homenaje a Héctor Fix Zamudio. México, D. F.: Editorial Porrúa

Ruiz Miguel, C. (1997). La ejecución de sentencias en el Tribunal Europeo de Derechos Humanos. Madrid: Editorial Tecnos.

Schwartz, B. (1966). Los poderes del Gobierno. (Vol. I). (J.J. Olloqui Labastida, Trad.) México, D. F.: Facultad de Derecho unAm.

Secretaría de Economía, Gobierno de México. (s.f.). Foro de Cooperación Económica AsiaPacífico (APEC). Recuperado de: http://www. economia.gob. $\mathrm{mx} /$ comunidad-negocios/comercio-exterior/organismos-multilaterales/ foro-de-cooperacion-economica-asia-pacificoapec

Secretaría de Economía, Gobierno de México. (2012). Memorias Documentales, Recuperado de: http://www.economia.gob.mx/files/ transparencia/informe_APF/memorias/12_ md_apec_sce.pdf

Soto Flores, A. (2012). Teoría de la Constitución. Supremacía constitucional. México, D. F.: Editorial Porrúa.

Suárez Camacho, H. (2007). El Sistema de Control Constitucional en México. México, D. F.: Editorial Porrúa. 
Suprema Corte de Justicia de la Nación. (s.f.). Reformas constitucionales en materia de amparo y derechos humanos. Recuperado de Suprema Corte de Justicia de la Nación: http:// www2.scjn.gob.mx/red/constitucion/

Suprema Corte de Justicia de la Nación. Segunda Sala. (Agosto, 1965). Tratados internacionales, amparo contra la aplicación de los. Tesis Aislada (Común). Semanario Judicial de la Federación, Sexta Época, Vol. XCIII, Tercera parte. Obtenido de scun: http://200.38.163.178/sjfsist/Paginas/DetalleGeneralV2.aspx?Epoca= $100000000 f 80000 \&$ Apendice $=100000000$ 0000\&Expresion $=$ TRATADOS $\% 2520$ INTERNA CIONALES\%2C\%2520AMPARO\%2520CONTR A\%2520LA\%2520APLICACI\%25C3\%2593N\%2520DE\%2520\%2520\%2520LOS\&Dominio $=$ Rubro,Texto\&TA_TJ=2\&Orden=1\&Clase $=\mathrm{D}$ etalleTesisBL\&NumTE=1\&Epp=20\&Desde $=-$ 100\&Hasta $=-100 \&$ Index $=0 \&$ InstanciasSelec cionadas $=6,1,2,3,4 \& \mid \mathrm{D}=265855 \& \mathrm{Hit}=1 \& \mathrm{IDs}$ $=265855 \&$ tipoTesis $=\&$ Semanario $=0 \&$ tabla $=$

Suprema Corte de Justicia de la Nación. Tribunal Pleno. (Diciembre 1992). Leyes federales y tratados internacionales. Tienen la misma jerarquía normativa. Tesis Aislada (Constitucional). Semanario Judicial de la Federación y su Gaceta, Octava Época, n. ${ }^{\circ}$ 60. Obtenido de scjn: http://200.38.163.178/sjfsist/ Paginas/DetalleGeneralV2.aspx?Epoca $=1 e 3$ e1fd00000000\&Apendice $=100000000000$ O\&Expresion=LEYES\%2520FEDERALES\%25 20Y\%2520TRATADOS\%2520INTERNACIONA LES.\%2520TIENEN\%2520LA\%2520MISMA\%
2520JERARQUIA\%2520NORMATIVA\&Domini $\mathrm{o}=$ Rubro,Texto \&TA_TJ=2\&Orden=1\&Clase $=\mathrm{D}$ etalleTesisBL\&NumTE $=2 \& E p p=20 \&$ Desde $=-$ $100 \&$ Hasta $=-100 \&$ Index $=0 \&$ InstanciasSelec cionadas $=6,1,2,3,4,5,50,7 \& \mid \mathrm{D}=205596 \& \mathrm{Hit}$ =2\&IDs=192867,205596\&tipoTesis=\&Sema nario $=0 \&$ tabla $=$

Suprema Corte de Justicia de la Nación. Pleno. (Abril 2007). Tratados internacionales. Son parte integrante de la Ley Suprema de la Unión y se ubican jerárquicamente por encima de las leyes generales, federales y locales. Interpretación del artículo 133 constitucional. Tesis Aislada (Constitucional). Semanario Judicial de la Federación y su Gaceta. Novena Época. Tomo XXV. Obtenido de scJN: http://200.38.163.178/sjfsist/Paginas/DetalleGeneralV2.aspx?Epoca $=1 \mathrm{e} 3 \mathrm{e} 10000000$ 000\&Apendice $=1000000000000 \&$ Expresio $\mathrm{n}=$ TRATADOS $\% 252$ INTERNACIONALES. $\% 25$ 20SON\%2520PARTE\%2520INTEGRANTE\%2 520DE\%2520LA\%2520LEY\%2520\%2520SU PREMA\%2520\%2520DE\%2520LA\%2520U NI\%25C3\%2593N\%2520Y\%2520SE\%2520 UBICAN\%2520JER\%25C3\%2581RQUICAME NTE\%2520POR\%2520ENCIMA\%2520DE\%2 520LAS\%2520LEYES\%2520GENERALES\% 2C\%2520FEDERALES\%2520Y\%2520LOCA LES.\%2520INTERPRETACI\%25C3\%2593N\% 2520DEL\%2520ART\%25C3\%258DCULO\%2 520133\%2520CONSTITUCIONAL\&Dominio $=$ Rubro, Texto \&TA_TJ=2\&Orden $=1 \&$ Clase $=\mathrm{D}$ etalleTesisBL $\&$ NumTE $=3 \& E p p=20 \&$ Desde $=-$ $100 \&$ Hasta $=-100 \&$ Index $=0 \&$ InstanciasSelec cionadas $=6,1,2,50,7 \&|\mathrm{D}=172650 \& \mathrm{Hit}=3 \&|$ 
Ds $=161358,167850,172650 \&$ tipoTesis $=\& S e$ manario $=0 \&$ tabla $=$

Suprema Corte de Justicia de la Nación. Tribunales Colegiados del Circuito. (Mayo 2010). Tratados internacionales. Cuando los conflictos se susciten en relación con derechos humanos, deben ubicarse a nivel de la Constitución. Tesis Aislada (Común). Semanario Judicial de la Federación, Novena Época, Tomo XXXI. Obtenido de scjn: http://200.38.163.178/ sjfsist/Paginas/DetalleGeneralV2.aspx?Ep oca $=1 \mathrm{e} 3 \mathrm{e} 10000000000 \&$ Apendice $=1000$ 000000000\&Expresion=TRATADOS $\% 2520$ INTERNACIONALES.\%2520CUANDO\%2520 LOS\%2520CONFLICTOS\%2520SE\%2520S USCITEN\%2520EN\%2520RELACI\%25C3\% 2593N\%2520CON\%2520DERECHOS\%25 2OHUMANOS\%2C\%2520DEBEN\%2520UBI CARSE\%2520A\%2520NIVEL\%2520DE\%25 20\%2520LA\%2520\%2520CONSTITUCI\%2 5C3\%2593N\&Dominio=Rubro,Texto\&TA_TJ= $2 \&$ Orden $=1 \&$ Clase $=$ DetalleTesisBL\&NumTE $=1 \& \mathrm{Epp}=20 \&$ Desde $=-100 \&$ Hasta $=-100 \& \mathrm{In}$ dex $=0 \&$ InstanciasSeleccionadas $=6,1,2,50$ ,7\&ID=164509\&Hit=1\&IDs=164509\&tipoTesis $=\&$ Semanario $=0 \&$ tabla $=$

Suprema Corte de Justicia de la Nación. (Junio de 2012). Reformas constitucionales en materia de amparo y derechos humanos. Obteni- do de Suprema Corte de Justicia de la Nación: http://www2.scjn.gob.mx/red/constitucion/

Suprema Corte de Justicia de la Nación. (24 de junio de 2011). Contradicción de Tesis 293/2011. "SCJN determina que las normas sobre derechos humanos contenidas en Tratados Internacionales tienen rango constitucional". Obtenido de scjn: http://www2.scjn. gob.mx/asuntosrelevantes/pagina/seguimientoasuntosrelevantespub.aspx?id=1296 59\&seguimientoid $=556$

Suprema Corte de Justicia de la Nación. Segunda Sala. (27 de junio de 2014). Control de constitucionalidad y convencionalidad. Condiciones para su ejercicio oficioso por los órganos jurisdiccionales federales. Jurisprudencia (Común). Semanario Judicial de la Federación, Décima Época. Obtenido de scsn: http:// sjf.scjn.gob.mx/SJFSem/Paginas/DetalleGeneralV2.aspx? Epoca $=\& A$ pendice $=\&$ Expresio $\mathrm{n}=\&$ Dominio=Tesis\%20\%20 publicadas $\% 20$ el\%20viernes\%2027\%20de\%20junio\%20 de\%202014.\%20Todo\&TA_TJ=1\&Orden$=3 \&$ Clase $=$ DetalleSemanarioBL $\&$ Tablero $=$ $\&$ NumTE $=13 \& E p p=20 \&$ Desde $=-100 \&$ Hast $a=-100 \&$ Index $=0 \&$ Semanald $=201426 \& \mid D=$ $2006808 \&$ Hit $=5 \& I D s=2006812,2006811$ - 